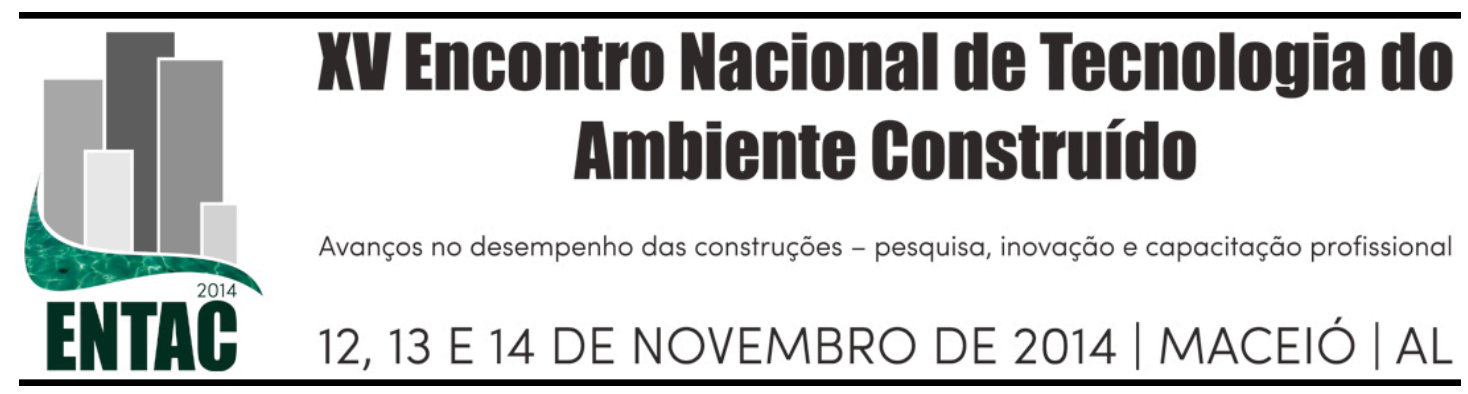

\title{
A AVALIAÇÃO DA CONSTRUÇÃO SUSTENTÁVEL NO BRASIL - MÉTODOS
}

\author{
UCHOA, Gabriela (1); MACÊDO, Lívia (2); BARTZ, Cíntia (3) \\ (1) UNINOVAFAPI, e-mail: gabrielauchoa@gmail.com, (2) UNINOVAFAPI, \\ e-mail: liviamaced@gmail.com, (3) UFPI, e-mail: cintiabartz@gmail.com
}

\begin{abstract}
RESUMO
A pesquisa sobre os métodos de classificação da sustentabilidade nas edificações surge num contexto recente de lançamento de selos de certificação de origem brasileira em contrapartida à aplicação de modelos de mensuração internacionais, como o amplamente difundido selo LEED - Leadership in Energy \& Environmental Design. A proposta deste artigo é debater a relevância da existência de selos nacionais de avaliação do grau de sustentabilidade de edificações, através dos quais podemos ter classificações condizentes com a realidade do país e adequada aos padrões de sustentabilidade nele desenvolvidos. Pretende também apresentar e discutir complexidade, abrangência e eficiência dos métodos utilizados. Em análise estão os selos AQUA e CASA AZUL e a etiqueta PROCEL EDIFICA, que através do método de pesquisas bibliográficas e junto aos órgãos responsáveis por cada, podemos constatar e entender os critérios considerados na avaliação. Através dessa pesquisa objetiva-se, além de explicar o funcionamento de cada método, divulgar suas finalidades, propósitos e vantagens. Procura-se também esclarecer a diversidade de tipologias construtivas que envolvem e a importância de cada uma destas no processo do desenvolvimento sustentável. A análise levou à conclusão de que o desenvolvimento destes selos nacionais de qualificação sustentável, além de baratearem os investimentos para a obtenção de certificação, também garantem uma classificação mais eficiente, pois são elaborados com base em informações sobre a indústria local, considerando diversidade climática e condições socioeconômicas existentes no País. Embora as três certificações (AQUA, PROCEL e CASA AZUL) venham para garantir medidas relativas à sustentabilidade, cada uma possui sua especificidade, verificando-se que uma não se sobrepõe à outra. Elas aparecem, na verdade, como opções de classificação que se adequarão mais ou menos à intenção da certificação.
\end{abstract}

Palavras-chave: Desenvolvimento Sustentável, Regulamentação, Desempenho.

\begin{abstract}
The research about the classification methods of sustainability in buildings arises in the context of the recent launch of certification label of Brazilian origin in contrast to the application of different international measurement, such as the widespread LEED - Leadership in Energy \& Environmental Design. The purpose of this article is to discuss the relevance of the existence of national labels assessing the level of sustainability of buildings, through which we may have consistent ratings with the reality of the country and the appropriate sustainability standards developed in it, and present and discuss complexity, scope and efficiency of the methods used. In the analysis are the following methods AQUA and CASA AZUL, and the label PROCEL EDIFICA, which by the method of bibliographic research and visiting institutions responsible for each, we can note and understand the criteria considered in the evaluation. Through this research we intend to not only explain the operation of each method as well as disclose goals, purposes and advantages. It also seeks to clarify the diversity of building typologies and the importance of involving each of these in the sustainable development process. The analysis led to the conclusion that the development of these national certification of sustainable construction, in addition to lower the investments for obtain certification, also ensures a more efficient classification, because its development focuses on local industry, climatic diversity and socioeconomic conditions in the country. Although the three certifications (AQUA, CASA AZUL and PROCEL) come to ensure measures related
\end{abstract}


the sustainability, each has its specificity, verifying that one does not override the other. They appear, in fact, as label options that will fit more or less the intent of the certification.

Keywords: Sustainable, Regulation, Performance. Development.

\title{
1 INTRODUÇÃO
}

Até a década de 70 o petróleo era a principal fonte de energia utilizada na indústria. A partir das crises econômicas, altas no preço do petróleo e a guerras nos países exportadores, causando grandes impactos na economia mundial, vários países desenvolveram estratégias para superar a dependência deste combustível, tais como: busca de fontes de energia alternativas e racionalização do consumo energético. A princípio, o grande motivador para estas transformações foi o fator financeiro, no entanto, posteriormente, foram adquirindo força as ideias de Consciência Ambiental, Aquecimento Global, Consumo Sustentável e a visão da necessidade da adoção de medidas que assegurassem um desenvolvimento sustentável e uma melhor qualidade de vida no futuro.

\begin{abstract}
O conceito de desenvolvimento sustentável foi introduzido pela primeira vez no Clube de Roma em 1968, como uma contestação ao modelo econômico adotado pelos países industrializados. Ali se introduziu a preocupação ambiental como necessária ao crescimento econômico (Wines, 2000). Ao longo do tempo, percebe-se que a visão de progresso (que se confundia com o domínio e transformação da natureza onde os recursos naturais eram vistos como ilimitados) e a preservação ambiental (que se limitava à criação de parques e áreas especiais destinadas à preservação de amostras da natureza para evitar a extinção de espécies) passaram a ser insuficientes (TRIANA et al., 2008).
\end{abstract}

Existem múltiplos conceitos para desenvolvimento sustentável, mas, de um modo comum, está presente neles a noção de que o desenvolvimento humano que ocorreu a partir da revolução industrial, alterou significativamente o equilíbrio ambiental do planeta. Além dos problemas ambientais, esse desenvolvimento trouxe consigo problemas socioeconômicos, como o aumento dos níveis de desigualdade tanto em escala local como global. Deste modo o desenvolvimento sustentável também deve estar envolvido com demandas sociais, constituindo-se, assim, no desafio de buscar o equilíbrio entre questões ambientais, sociais e econômicas - proteção, justiça e viabilidade (CAIXA, 2010).

Nos últimos anos foram realizados debates e convenções, em esfera nacional e internacional, para discussão do tema Sustentabilidade, onde metas foram estipuladas para um consumo racional dos recursos naturais e para a redução do impacto que a ação humana gera ao meio ambiente. Foi em meio a essas discussões que se verifica a responsabilidade do setor da Construção Civil como um dos maiores consumidores de recursos naturais. Este torna-se, portanto, um dos principais focos de proposição de mudanças na luta por um desenvolvimento sustentável (MARQUES e SALGADO, 2007).

Estima-se que os resíduos gerados pela construção civil sejam em torno de $500 \mathrm{gh} / \mathrm{hab}$. por ano (CAIXA, 2010), e que estes são, em sua maioria, depositados em locais inadequados. Apesar dos esforços para conscientização deste problema, a construção civil ainda é, provavelmente, o maior gerador de resíduos da economia. Assim, para o desenvolvimento sustentável é necessário que se construa de forma eficiente, ou seja, construindo mais utilizando menos matéria prima, reduzindo desperdícios e prevendo sempre que possível a reutilização de resíduos no lugar de matéria extraída da natureza. 
No Brasil, a preocupação com a construção sustentável foi mais incisiva a partir da década de 90, quando foram realizados estudos sistemáticos para a redução de resíduos, perdas e energia, assim como para reciclagem. Nesse mesmo período, Loura et al. (2009) destaca que a crise energética acontecida no Brasil, cujo resultado foi o racionamento de energia elétrica ocorrido no ano de 2001, também contribuiu para que o governo brasileiro voltasse seus olhos para o uso racional da energia elétrica, em especial nas edificações. Para adotar as medidas necessárias para avaliação da eficiência energética, foram desenvolvidos os selos de certificação de construção sustentável nacionais.

Dentre estes, destacam-se as certificações AQUA, a etiqueta PROCEL EDIFICA e selo CASA AZUL. No entanto, a recente aplicação destes selos ainda não permite afirmações sobre a eficiência de seus critérios para a construção de edificações realmente sustentáveis. Deste modo, o que se permite nesta pesquisa é o estudo comparativo destes e dos seus critérios de pontuação, para analisar como e com qual profundidade estes contemplam questões fundamentais para o desenvolvimento sustentável, não apenas questões energéticas, como também fatores sociais e econômicos.

\section{A AVALIAÇÃO DA SUSTENTABILIDADE NA CONSTRUÇÃO BRASILEIRA - CERTIFICAÇÕES NACIONAIS}

A avaliação da adoção de práticas sustentáveis na construção funciona como um atestado que visa informar à sociedade que as edificações estão de acordo com parâmetros para redução de consumo de energia e água e conforto ambiental. A necessidade do estabelecimento de critérios de avaliação vem de fontes de pressão distintas, entre elas a regulamentação governamental, a necessidade de reação face aos impactos ambientais e a própria demanda do mercado. A sociedade cada vez mais consciente e informada força a cobrança pelo desenvolvimento sustentável e pressiona as empresas a reverem e atestarem suas práticas.

De início, no Brasil, estabeleceram-se os critérios de mensuração internacional, sendo amplamente difundido o selo de origem americana LEED - Leadership in Energy \& Environmental Design, sob a responsabilidade do Green Building Council Brasil - GBC Brasil. No entanto, faz-se necessária a compreensão do contexto da edificação para a correta avaliação das medidas para sustentabilidade, conhecer o funcionamento da indústria nacional, seus condicionantes e impactos são determinantes para que as decisões do projeto não sejam tomadas por ideias pré-concebidas, por isso é importante a pesquisa de padrões locais (CBIC, 2012).

Neste esforço, o Brasil vem apresentando alternativas para a avaliação das edificações, não existindo ainda um método amplamente difundido e utilizado, de modo que cada construtor busca a certificação que lhe for mais conveniente. Faz-se necessário o estudo destes classificadores, para que haja uma maior reflexão sobre seus resultados e impactos reais na melhoria dos critérios de sustentabilidade na construção.

\subsection{Selo AQUA}

A certificação AQUA - Alta Qualidade Ambiental - foi o primeiro selo criado no Brasil e é hoje considerado o mais importante dentre os nacionais, tendo certificado mais de 40 edificações no país de diferentes categorias ${ }^{1}$. O selo foi lançado em 2008, pela

\footnotetext{
${ }^{1}$ Segundo dados obtidos no site da Fundação Carlos Alberto Vanzolini, em junho de 2012.
} 
Fundação Carlos Alberto Vanzolini, em parceria com entidades pesquisadoras como a Escola Politécnica da Universidade de São Paulo e o Centre Scientifique et Technique du Bâtiment (CSTB), sendo baseado em normas europeias, sobretudo o selo francês HQE (Haute Qualité Environmentale), porém tendo seus indicadores adequados à realidade brasileira.

O diferencial deste selo é a intenção de avaliar a eficiência das medidas adotadas em diferentes fases do empreendimento - programa, concepção, realização e operação, e medi-las de forma qualitativa e não quantitativa, não apenas na fase de projeto, como também são feitas auditorias na fase construtiva para aferição das escolhas. Assim, ao contrário de se atribuir um valor numérico para o atendimento de determinados critérios, é atribuída uma classificação para o atendimento dos requisitos, que uma vez atendidos podem ser considerados bom, superior ou excelente, conforme a eficiência do item em questão (FCAV, 2007).

Ao todo são avaliados 14 requisitos dentro de 4 parâmetros, sendo eles:

1 Eco Construção: Relação do edifício com seu entorno; Escolha integrada de produtos, sistemas e processos construtivos; Canteiro de Obras com baixo impacto ambiental.

2 Gestão: Energia; Água; Resíduos de uso e operação do edifício;

3 Manutenção - permanência do desempenho ambiental: Conforto; Higrotérmico; Acústico; Visual; Olfativo.

4 Saúde: Qualidade sanitária dos ambientes; Qualidade Sanitária do Ar; Qualidade Sanitária da Água.

Enquanto certificações como o LEED e o BREAM se fundamentam no somatório de pontos, o selo AQUA qualifica os itens exposto acima, atribuindo um juízo que o coloca entre os três níveis abaixo:

- Bom: desempenho mínimo aceitável para um empreendimento de Alta Qualidade Ambiental;

- Superior: apresenta boas práticas de sustentabilidade;

- Excelente: corresponde ao desempenho máximo constatado em empreendimentos de Alta Qualidade Ambiental.

Para obter o atestado, é preciso que sejam atendidos no mínimo três critérios com a qualificação excelente, e, no máximo, que sete deles sejam considerados somente bom. $\mathrm{O}$ atendimento destes valores mínimos (três excelente, quatro superior e sete bom) enquadrará o empreendimento no perfil mínimo para que se receba o certificado.

As vantagens deste sistema em relação a outros aplicados no Brasil são percebidas tanto para o empreendedor como para a sociedade. Para o empreendedor porque os critérios estabelecidos como medidas sustentáveis são mais adaptados às condições ambientais e socioeconômicas do Brasil, é mais flexível e avalia a eficiência das escolhas tomadas. $\mathrm{O}$ ganho da sociedade está na construção com redução do consumo de matéria prima, da geração de resíduos e da poluição, que é verificado também após a entrega da obra, com monitoramento contínuo.

Uma das limitações do AQUA é que possui referencial técnico apenas para edifícios habitacionais, bairros, loteamentos e do setor de serviço tais como edifícios de escritório e escolares. No entanto, existe a possibilidade para o cliente de, caso seu 
empreendimento não possua referencial técnico disponível, este pode servir de piloto a partir de um referencial básico, para que se testem as adaptações e exigências necessárias para receber a certificação, quando passará a servir de referência para as demais construções daquele setor.

\subsection{Etiqueta PROCEL EDIFICA}

A etiqueta de avaliação PROCEL EDIFICA é um dos subprogramas do Programa Nacional de Conservação de Energia Elétrica - PROCEL, criado em dezembro de 1985, pelo Ministério de Minas e Energia e da Indústria e Comércio. A criação do programa, de iniciativa do Governo Federal, teve como objetivo combater o desperdício e reduzir os custos e investimentos no setor energético, estimulando a produção de bens que utilizasse a energia de modo eficiente.

Em 1993, foi instituído a Etiqueta Procel de Economia de Energia, que indica ao consumidor, ao comprar um bem, quais os produtos que apresentam o melhor desempenho no consumo de energia em cada categoria. Assim estimula o consumo, a fabricação e comercialização de produtos mais eficientes, reduzindo assim custo na geração de energia e impactos ambientais (CARVALHO, 2012).

Em 2003, teve início o programa de expansão desta etiquetagem para o setor da construção civil, com a elaboração do Plano de Ação do Procel Edifica, no entanto, apenas em 2009 foi lançado oficialmente para avaliação de edifícios comerciais e públicos, como parte do Programa Brasileiro de Etiquetagem. Em novembro de 2010 foi lançada a versão para avaliação de edificações residenciais. Este programa de etiquetagem é resultado da parceria entre a Centrais Elétricas Brasileiras S.A. Eletrobrás - e o Instituto Nacional de Metrologia, Qualidade e Tecnologia - Inmetro. A outorga da etiqueta é conferida pelo Laboratório de Eficiência Energética em Edificações - LabEEE, da Universidade Federal de Santa Catarina (LAMBERTS et al., 2007).

A concessão da etiqueta PROCEL EDIFICA pode ser conferida em duas ocasiões, podendo ser na fase de projeto ou após edificação construída, o que irá influenciar o método de análise. Na primeira situação é aplicado o método prescritivo ou o método da simulação, enquanto a edificação já executada deverá ser avaliada através de inspeção presencial.

Os critérios de avaliação também irão diferir de acordo com a categoria do empreendimento. As edificações públicas ou destinadas a comércio e serviços tem sua avaliação dividida em três requisitos, sendo eles (1) eficiência e potência instalada do sistema de iluminação, (2) eficiência do sistema de condicionamento do ar e (3) desempenho técnico da envoltória do edifício. Os níveis de eficiência destes itens são classificados em uma escala que varia de A (mais eficiente) a $\mathrm{E}$ (menos eficiente).

$\mathrm{Na}$ obtenção da classificação geral do empreendimento, os três critérios são rotulados, onde cada requisito possui um peso para a pontuação final, onde $30 \%$ corresponde ao sistema de iluminação, outros $30 \%$ à envoltória e $40 \%$ corresponde ao sistema de condicionamento de Ar. Existe ainda a bonificação de pontos para as edificações que apresentem sistemas ou fontes renováveis de energia, sistemas de cogeração de energia e uso racional da água, que colaboram para o aumento em um nível na classificação geral do edifício. Na avaliação PROCEL EDIFICA é possível ainda obter a certificação parcial para as edificações de caráter público, comercial ou de prestação de serviços, ou seja, em apenas um dos critérios de avaliação (MME, 2008). 
Já nas edificações residenciais são avaliados a envoltória e o sistema de aquecimento de água. Neste caso só poderá ser avaliado o desempenho do edifício como um todo, considerando o desempenho do edifício no verão quando naturalmente ventilado ou desempenho no inverno para as regiões de clima mais frio no país.

Além destes critérios, são também considerados pré-requisitos mínimos para que as construções obtenham classificação de nível A. Uma delas é a utilização de fontes de energia alternativa para o aquecimento de água se houver demanda (aquecimento solar, aquecedores a gás, etc), instalação de controle inteligente de tráfego para edificações com mais de um elevador e uso de bombas e água centrífuga participantes do Programa Brasileiro de Etiquetagem.

O processo de outorga da etiqueta do PROCEL é constituído por duas etapas. A primeira consiste na verificação do projeto e da documentação do empreendimento, onde se emite um certificado com a etiqueta que atesta o nível da eficiência do projeto. Já na segunda se realiza a auditoria da edificação em uso, onde já estará habitado e com os sistemas instalados, onde, se este corresponder ao desempenho esperado, receberá uma placa com o certificado para ser exposta no edifício.

A etiqueta PROCEL para a construção civil apresenta-se, então, como uma ferramenta prática para a comparação de edificações quando se tem em questão o desempenho na redução do consumo de energia elétrica, não abrangendo outros critérios considerados nas construções sustentáveis.

\subsection{Selo CASA AZUL}

Diferente da certificação da Eletrobrás, o Selo Casa Azul foi criado com a proposta de englobar em sua avaliação diversas categorias e subcategorias que tornam uma edificação sustentável, envolvendo não apenas aspectos como eficiência energética, como também a qualidade urbana, questões sociais, gestão de recursos, etc. A criação do selo parte de um conjunto de ações da Caixa Econômica Federal - CAIXA - que objetivavam integrar ações voltadas para a consciência de um desenvolvimento sustentável nos produtos oferecidos pelo banco como política que atesta a responsabilidade social da instituição.

Como responsável por cerca de $70 \%$ do crédito imobiliário para financiamento de projetos residenciais no Brasil, a CAIXA tem papel importante na indução de práticas a serem adotadas na construção de empreendimentos habitacionais (CAIXA, 2010). Consciente desta função, a CAIXA veio a lançar o selo em 2010, sendo desenvolvido, ainda, apenas para a classificação de projetos de habitação vinculados aos programas de financiamentos ou repasse da instituição, tais como o "Minha Casa, Minha Vida". O selo é de caráter voluntário e funciona como um incentivo ao reconhecer as boas práticas das empresas para se produzir construções mais sustentáveis.

O Selo Casa Azul CAIXA é um instrumento de classificação socioambiental de projetos de empreendimentos habitacionais, que busca reconhecer os empreendimentos que adotam soluções mais eficientes aplicadas à construção, ao uso, à ocupação e à manutenção das edificações, objetivando incentivar o uso racional de recursos naturais e a melhoria da qualidade da habitação e de seu entorno. (CAIXA, 2010).

O método desenvolvido pela CAIXA funciona verificando, durante a análise de viabilidade técnica do empreendimento para atendimento aos programas de financiamento, o atendimento aos requisitos exigidos pelo selo. Além dos critérios de avaliação, a instituição exige que todos os projetos que se candidatem à obtenção do 
CASA AZUL atendam às regras da Ação Madeira Legal e apresentar, até o fim da obra, o Documento de Origem Florestal e declaração informando volume, espécie e destinação final das madeiras utilizadas. Quanto aos critérios estabelecidos pelo selo, ao todo são 53, divididos em 6 categorias que irão nortear a classificação do projeto.

A categoria 1 - Qualidade Urbana - compreende as ações que remetem sobretudo à escolha do sítio e a relação entre empreendimento, entorno natural, construído e comunidade, de forma a se otimizar o uso das infraestruturas existentes, o desenvolvimento das comunidades existentes e a criação de comunidades atraentes, com aumento da qualidade de vida no local. Nesta categoria é obrigatório o atendimento aos critérios de melhoria da qualidade de vida, considerando a existência de infraestrutura do entorno e de busca de bem estar, segurança e saúde para os moradores.

Os requisitos relacionados ao projeto e ao conforto das edificações são contemplados na categoria 2 , onde tenciona que os projetos considerem as questões relativas à adaptação das edificações às características climáticas, físicas e geográficas locais, bem como relativas à destinação de espaço para atividades específicas.

Os requisitos relacionados à eficiência energética são englobados na categoria 3, onde é indispensável a adoção das práticas de utilização de lâmpadas de baixo consumo nas áreas privativas, dispositivos economizadores nas áreas comuns e medição de gás individualizada para se obter a certificação. Na categoria 4, de Conservação de Recursos Materiais, é imprescindível que sejam atendidas as condições de qualidade de materiais e componentes, de utilização de formas e escoras reutilizáveis, e de gestão de resíduos de demolição e construção.

Ainda envolvendo a gestão de recursos, a categoria 5 compreende a gestão da água, onde adota políticas para mitigar problemas de escassez e poluição, bem como redução de riscos de inundação nas cidades. Por fim, no conjunto de ações para a sustentabilidade nas edificações habitacionais, tem-se a categoria destinada à avaliação das práticas sociais, categoria 6 , onde pretende-se promover ações que integrem os diversos agentes envolvidos na elaboração do projeto, construção e utilização das edificações, entendendo que todos têm responsabilidade socioambiental em seu envolvimento com o empreendimento. Neste grupo são obrigatórias as práticas de educação para a gestão de Resíduos de Construção e Demolição, de educação ambiental dos empregados e de orientação dos moradores.

Para a obtenção do selo é necessário que se atenda pelo menos os requisitos obrigatórios, mas é a adesão ao maior número de indicadores que elevará o nível da certificação dos empreendimentos, que podem ser qualificados como Ouro (critérios obrigatórios +12 itens de livre escolha), Prata (critérios obrigatórios +6 itens de livre escolha) ou Bronze (critérios obrigatórios) conforme listado abaixo:

1 Qualidade Urbana: Qualidade do Entorno - Infraestrutura ${ }^{2}$, Qualidade do Entorno - Impactos ${ }^{2}$, Melhorias no Entorno, Recuperação de Áreas Degradadas, Reabilitação de Imóveis.

2 Projeto e Conforto: Paisagismo ${ }^{2}$, Flexibilidade de Projeto, Relação com a Vizinhança, Solução Alternativa de Transporte, Local para Coleta Seletiva ${ }^{2}$, Equipamentos de Lazer, Sociais e Esportivos ${ }^{2}$, Desempenho Térmico Vedações ${ }^{2}$, Desempenho Térmico - Orientação ao Sol e Ventos ${ }^{2}$, Iluminação 
Natural de Áreas Comuns, Ventilação e Iluminação Natural de Banheiros, Adequação às Condições Físicas do Terreno.

3 Eficiência Energética: Lâmpadas de Baixo Consumo - Áreas Privativas ${ }^{3}$, Dispositivos Economizadores - Áreas Comuns ${ }^{2}$, Sistema de Aquecimento Solar, Sistemas de Aquecimento à Gás, Medição Individualizada - Gás ${ }^{2}$, Elevadores Eficientes, Eletrodomésticos Eficientes, Fontes Alternativas de Energia.

4 Conservação de Recursos Materiais: Coordenação Modular, Qualidade de Materiais e Componentes ${ }^{2}$, Componentes Industrializados ou Pré-fabricados, Formas e Escoras Reutilizáveis ${ }^{2}$, Concreto com Dosagem Otimizada, Cimento de Alto-Forno (CPIII) e Pozolânico (CP IV), Pavimentação com Resíduos de Construção e Demolição - RCD, Facilidade de Manutenção da Fachada, Madeira Plantada ou Certificada.

5 Gestão da Água: Medição Individualizada - Água ${ }^{2}$, Dispositivos Economizadores - Sistema de Descarga, Dispositivos Economizadores Arejadores, Dispositivos Economizadores - Registro Regulador de Vazão, Aproveitamento de Águas Pluviais, Retenção de Águas Pluviais, Infiltração de Águas Pluviais, Áreas Permeáveis²

6 Práticas Sociais: Educação para a Gestão de RCD, Educação Ambiental dos Empregados, Desenvolvimento Pessoal dos Empregados, Capacitação Profissional dos Empregados, Inclusão de trabalhadores locais, Participação da Comunidade na Elaboração do Projeto, Orientação aos Moradores ${ }^{2}$, Educação Ambiental dos Moradores, Capacitação para Gestão do Empreendimento, Ações para Mitigação de Riscos Sociais, Ações para a Geração de Emprego e Renda.

Como diferencial para a obtenção deste selo, a caixa não cobra pela sua concessão, sendo onerosa apenas a taxa de análise de projeto, que não deve ultrapassar o teto de $\mathrm{R} \$ 328,00$. O baixíssimo custo desta avaliação tem a função de tornar atraente a adesão das empresas ao programa, enquanto, do outro lado, os empreendimentos ganham valor agregado por serem considerados sustentáveis e empresas obtêm reconhecimento da sociedade pelo investimento em práticas sociais e ambientalmente corretas.

No entanto, apesar da complexidade do programa da CAIXA, deve-se ressaltar que se trata de um programa limitado, onde são analisados apenas os projetos vinculados aos programas de financiamento do banco, e, assim, não pode concorrer ou ter caráter efetivo de uma certificação.

\section{VANTAGENS E DESVANTAGENS DOS SISTEMAS}

Visto as limitações de propósitos e fins específicos dos métodos de avaliação discutidos, não se faz razoável estabelecer um comparativo entre os diferentes caminhos de avaliação, uma vez que possuem objetivos diferenciados. No entanto, o que deve ser observado na adesão por determinado programa é quais as características do empreendimento que alinham o propósito de suas práticas sustentáveis ao de determinada certificação.

Quando se tem o intuito de se obter uma certificação de padrão internacional, que sejam equiparadas a sistemas estrangeiros como HQE, LEED ou BREEAM, no entanto adaptados à realidade brasileira, provavelmente a opção deverá ser pelo sistema AQUA, 
pois envolve um conjunto de medidas que tem se consolidado como práticas adequadas, abrangendo diversas formas de atuação no campo da construção sustentável.

O selo AQUA distingue-se pela sua avaliação qualitativa dos indicadores e possibilita ao empreendedor a escolha das medidas que comprovar eficientes no atendimento destes critérios. No entanto, a complexidade de etapas e exigências, associado ao elevado custo de sistemas como este ainda limitam seu uso no país, sobretudo nas áreas de menor desenvolvimento econômico, onde ainda persistem práticas já superadas na construção civil sustentável.

Torna-se assim, mais atraente a opção por selos como o CASA AZUL, onde a verificação do atendimento a certa quantidade de requisitos classificam a construção como sustentável, ou seja, por método quantitativo. Contudo, como explicitado, as barreiras de utilização destes sistemas estão no condicionamento da análise ao atrelamento à relação instituição financeira-cliente, bem como à referência somente de edificações habitacionais.

Apesar desta limitação, reconhece-se a importância do selo, pois o seu baixo custo de avaliação e a clareza das medidas a serem adotadas estimulam empresas a se candidatarem para obter a classificação. Outro ponto que merece ser ressaltado é a inserção de requisitos que consideram medidas que favoreçam a transformação social e a diminuição de desigualdades.

A etiqueta PROCEL, se comparado a outros mecanismos de medida dos índices de sustentabilidade das construções, tem objetivos mais específicos. Não pretende mensurar o desempenho dos edifícios em face de um envolvimento geral para um desenvolvimento sustentável, e sim atuar como um indicador da eficiência energética. Oferecendo uma ferramenta comparativa prática para que o usuário escolha por uma ou outra edificação. Esta avaliação simplificada e de fácil identificação é importante para a fácil assimilação do usuário e possibilitar assim seu uso em larga escala, podendo tornar-se obrigatório para todas as edificações nacionais.

\section{CONCLUSÕES}

A princípio, para se avaliar o grau de sustentabilidade das edificações no Brasil, tinha-se que recorer aos selos internacionais que não se adequavam de todo à realidade da construção civil no país. Ao se desenvolverem certificados nacionais de qualificação sustentável, além de baratearem os investimentos para a obtenção destes selos, também garante-se uma classificação mais eficiente, já que são elaborados com base em informações sobre a indústria local, levando em consideração a diversidade climática e as condições socioeconômicas existentes no País.

Embora as três certificações (AQUA, PROCEL e CASA AZUL) venham para garantir medidas relativas à sustentabilidade, cada uma possui sua especificidade e, portanto, nenhuma se sobrepõe à outra. Elas aparecem na verdade como opções de certificação mais adequada ao empreendimento em questão. Se levarmos em conta o investimento para obter a classificação de habitações populares, por exemplo, o selo que mais se encaixa é o CASA AZUL, por seu baixo custo. Para a adoção em larga escala, visando o barateamento do consumo de energia elétrica, o PROCEL EDIFICA se mostra o melhor por ter um processo mais simplificado, surgindo como um programa de governo para a redução dos custos nacionais com a produção de energia. Já o AQUA é o único que analisa além das fases de concepção do projeto e execução; indo além garantindo a certificação em relação à operação dos mecanismos instalados, certificando a eficiência 
dos mesmos e assegurando a permanência do desempenho ambiental ao longo do seu uso.

Apesar disso, principalmente por serem certificações recentes, nenhuma delas garantem um desempenho sustentável. Elas proporcionam condições técnicas para que o edifício possua potencial para um bom desempenho, mas o que vai de fato garantir que esse desempenho aconteça são seus futuros usuários e responsáveis pela operação dos mecanismos. Assim percebe-se a importância de se trabalhar a educação social com orientações e conscientização para que o comportamento humano não venha a alterar ou subutilizar a capacidade das edificações, sejam elas certificadas ou não.

\section{REFERÊNCIAS}

CAIXA ECONÔMICA FEDERAL - CAIXA. Boas práticas para habitação mais sustentável / coordenadores Vanderley Moacyr John, Racine Tadeu Araújo Prado. São Paulo : Páginas \& Letras - Editora e Gráfica, 2010.

CARVALHO, V. Métodos de classificação quanto a eficiência energéticas em edificação e estudo de caso. Monografia. UFRJ. Rio de Janeiro, 2011. Disponível em: $<$ http://www.monografias.poli.ufrj.br/monografias/monopoli10003945.pdf $>$. Acesso em junho de 2012.

CBIC. Construção Verde: Desenvolvimento com sustentabilidade / Confederação Nacional da indústria. Câmara brasileira da indústria da construção. - Brasília: CNi, 2012.

FUNDAÇÃO CARLOS ALBERTO VANZOLINI - FCAV. Referencial técnico de certificação - edifícios do setor de serviço- processo AQUA. Francisco Cardoso (Coordenação). FCAV. São Paulo, 2007. Disponível em: <http://vanzolini.org.br/download/RTEscritorios\%20e\%20Edif\%C3\%ADcios\%20escolares-V0-outubro2007.pdf>. Acesso em junho de 2012.

LAMBERTS, R; CARLO, J., GOULART, S.; WESTPHAL F. Regulamentação de etiquetagem voluntária de nível de eficiência energética de edifícios comerciais e Públicos. In: IX Encontro Nacional de Conforto do Ambiente Construído, anais... : ENCAC, Ouro Preto. 2007.

LOURA, R. et al. Status e discussão da regulamentação de eficiência em edificações brasileira. In: III Congresso Brasileiro de Eficiência Energética, anais eletrônicos... Belém, 2009. Disponível em: http://www.aresarquitetura.com.br/downloads/CBEE2009.pdf. Acesso em junho de 2012.

MARQUES,F; SALGADO, M. Padrões de Sustentabilidade aplicados ao processo de projeto. In: VII Workshop Brasileiro de Gestão do Processo de Projeto na Construção de Edifícios, anais eletrônicos... Curitiba, 2007. Disponível em: $<$ http://www.cesec.ufpr.br/workshop2007/Artigo-16.pdf $>$. Acesso em: julho de 2012.

MINISTÉRIO DAS MINAS E ENERGIA - MME. Etiquetagem de Eficiência Energética de Edificações. 04 volumes. Brasília, 2008. Disponível em: <www.labeee.ufsc.br/eletrobras> Acesso em julho de 2012.

TRIANA, A.; LAMBERTS, R.; FOSSATI, M.; MATISTA, J. Sustentabilidade nas Edificações: contexto internacional e algumas referências brasileiras na área. Disponível $\mathrm{em}:<\mathrm{http}: / /$ www.labeee.ufsc.br/sites/default/files/documents/sustentabilidade_nas_edificacoes_c ontexto_internacional_e_algumas_referencias_brasileiras_na_areasustentabilidade_nas_edificac oes_contexto_internacion>. Acesso em: julho de 2012. 